\title{
Facilitación y disminución condicionada de la respuesta incondicionada en humanos
}

\section{Conditioned Diminution and Facilitation of the Unconditioned Response in Humans facilitação e diminuição condicionada da resposta incondicionada em humanos}

\author{
Sebastián Agustín Becerra Céspedes, Jorge Andrés Pinto Pinto* \\ Universidad de Talca
}

Doi: dx.doi.org/10.12804/apl33.01.2015.01

\section{Resumen}

La disminución condicionada de la respuesta incondicionada es un fenómeno de priming, según el cual los estímulos predichos o anticipados tienden a ser procesados con menor efectividad que cuando son inesperados. Esta hipótesis fue examinada por medio de un experimento en el cual se sometió a un grupo de estudiantes a una sesión de condicionamiento donde recibieron un total de 132 ensayos consistentes en la presentación de estímulos auditivos y vibraciones. De estos ensayos, 66 correspondían al Estímulo Condicionado Reforzado $(\mathrm{EC}+)$ que co-termina con un soplo de aire como Estímulo Incondicionado (EI), entremezclados con 66 ensayos del Estímulo Condicionado no Reforzado (EC-) por sí solo. Posteriormente, en una sesión de prueba los participantes recibieron una serie de ensayos en los cuales se examinó la RI a estímulos incondicionados de alta y baja intensidad cuando eran precedidos por los $\mathrm{EC}+\mathrm{y}$ EC-. Los resultados arrojaron una mayor amplitud de la Respuesta Condicionada (RC) al EC+ en comparación con el EC- en el curso del entrenamiento y una mayor amplitud de la Respuesta Incondicionada (RI) al EC+ cuando los ECs fueron probados con un EI de baja intensidad, mientras que, contrario a lo esperado, no hubo diferencias significativas en la amplitud de la RI cuando los ECs fueron probados con un EI de alta intensidad. Estos hallazgos no son concluyentes para determinar que el estímulo condicionado tiene dos formas opuestas de influir en la magnitud de la respuesta incondicionada. Palabras clave: Condicionamiento, facilitación condicionada, disminución condicionada

\section{Abstract}

Conditioned diminution of the unconditioned response is a phenomenon of priming, in which the predicted or anticipated stimuli tend to be processed less effectively than when they are unexpected. This hypothesis was examined in an experiment in which a group of students received a conditioning session consisting of the presentation of a total of 132 trials of auditory and vibration stimuli, of which 66 trials corresponded to Conditioned Stimuli with Reinforcement (CS+) that co-terminated

\footnotetext{
* Sebastián Agustín Becerra Céspedes, Universidad de Talca; Jorge Andrés Pinto Pinto, Universidad de Talca. Agradecemos a Fernando Ponce y Edgar Vogel por su colaboración en la investigación desarrollada.

La correspondencia relacionada con este artículo debe ser dirigida a Jorge Andrés Pinto Pinto, Universidad de Talca, Facultad de Psicología, Talca, Chile. Correo electrónico: jpinto.utalca@gmail.com
} incondicionada en humanos. Avances en Psicología Latinoamericana, 33(1), 1-14. doi: dx.doi.org/10.12804/ap133.01.2015.01 
with an air puff as Unconditioned Stimulus (US), interspersed with 66 trials of Conditioned Stimuli without Reinforcement (CS-) alone. Subsequently, in a testing session, participants received a series of trials in which the unconditioned response (UR) was examined to unconditioned stimuli of high and low intensity when the stimulus was preceded by CS+ or CS-. The results showed greater amplitude of CR to the CS+ compared to the CS- during training and greater amplitude of the UR to the CS + when the CSs were tested with US low intensity while contrary to expectations, there were no significant differences in the amplitude of the UR when CSs was tested with high intensity USs.

These findings are not conclusive as to whether the conditioned stimulus has two opposite ways to influence the magnitude of the unconditioned response.

Key words: Conditioning, conditioned facilitation, conditioned diminution

\section{Resumo}

A diminuição condicionada da resposta incondicionada é um fenómeno de "priming", segundo o qual os estímulos preditos ou antecipados tendem a ser processados com menor efetividade que quando são inesperados. Esta hipótese foi examinada através de um experimento no qual se submeteu a um grupo de estudantes a uma sessão de condicionamento onde receberam um total de 132 ensaios consistentes na apresentação de estímulos auditivos e vibrações, dos quais 66 ensaios correspondiam ao estímulo $\mathrm{EC}+$ que termina com um sopro de ar como EI, entremeados com 66 ensaios do estímulo EC- por si só. Posteriormente, em uma sessão de prova os participantes receberam uma série de ensaios nos quais examinou-se a RI a estímulos incondicionados de alta e baixa intensidade quando eram precedidos pelos estímulos EC+ y EC-. Os resultados expuseram uma maior amplitude da $\mathrm{RC}$ ao EC+ em comparação ao EC-no curso do treinamento e uma maior amplitude da RI ao EC+ quando os ECs foram provados com um EI de baixa intensidade enquanto que contrário ao esperado, não houve diferenças significativas na amplitude da RI quando os ECs foram provados com um EI de alta intensidade.

Estes achados não são concluintes para determinar que o estímulo condicionado tem duas formas opostas de influir na magnitude da resposta incondicionada.

Palavras-chave: Condicionamento, facilitação condicionada, diminuição condicionada

Tras sucesivos emparejamientos de un estímulo conductualmente neutral, denominado Estímulo Condicionado (EC), con uno biológicamente significativo o Estímulo Incondicionado (EI), el EC adquiere la capacidad de generar una Respuesta Condicionada (RC). Este tipo de aprendizaje se conoce como condicionamiento clásico (Pavlov, 1927).

Un procedimiento que satisface particularmente bien las demandas actuales es el condicionamiento de parpadeo en conejos (Gormezano, Schneiderman, Deux \& Fuentes, 1962). En este procedimiento, los conejos, quienes raramente parpadean, aprenden a parpadear a estímulos visuales, auditivos y táctiles después de emparejarlos con un soplo de aire en la córnea o con una estimulación eléctrica en los músculos oculares. Uno de los atractivos de este procedimiento es la robustez y nitidez con la que se produce la aparición progresiva de una respuesta condicionada de parpadeo, la cual posee una sorprendente precisión temporal, al iniciarse en el momento justo en el que se espera que ocurra el estímulo incondicionado. Así, después de una gran cantidad de emparejamientos entre el EC y el EI, los conejos no solo aprenden a anticipar el EI emitiendo una respuesta condicionada, sino que también aprenden, con extraordinaria exactitud, el momento en el que deben emitir esta respuesta. Esto ha motivado a los investigadores a formular modelos matemáticos sofisticados de condicionamiento que tengan en cuenta no solo la generación de la respuesta sino también su topografía temporal. 
Por otra parte, los modelos matemáticos inspirados en el procedimiento de condicionamiento de parpadeo en conejos han motivado la búsqueda de sus correlatos neurobiológicos, encontrándose evidencia de participación de estructuras tales como el cerebelo, la amígdala y el hipocampo. Por ejemplo, se han encontrado las vías neuronales que están involucradas en el procesamiento conjunto del EC y el EI en el núcleo del cerebelo (Christian \& Thompson, 2003; Thompson, Thompson, Kim, Krupa \& Shinkman, 1998) y los sitios que explicarían el ajuste temporal de la respuesta en sinapsis plásticas de las células Golgi en la corteza cerebelar (Mauk, Medina, Nores \& Ohyama, 2000). Además, se ha determinado que el hipocampo cumpliría un rol central en la retención de los estímulos en la memoria de corto plazo cuando estos ya no se encuentran presentes (Sanderson, Pearce, Kyd \& Aggleton, 2006) y la amígdala actuaría como un agente modulador de la respuesta condicionada de parpadeo por medio de un sistema paralelo de condicionamiento emocional (Fanselow \& LeDoux, 1999; Lanuza, Moncho-Bogani \& LeDoux, 2008).

Con estos datos neurobiológicos en la mano, los investigadores han especulado acerca de las implicaciones de estos hallazgos para la neurobiología del aprendizaje en humanos (Steinmetz, Tracy \& Green, 2001). Por ejemplo, se han sugerido relaciones entre el déficit de condicionamiento y ciertas patologías del cerebelo e hipocampo (Daum et al., 1993). Las investigaciones en esta materia se han realizado con participantes que padecen amnesia (Schugens \& Daum, 1999), lesiones cerebelares diversas (Bracha, Zhao, Wunderlich, Morrissy \& Bloedel, 1997), autismo (Sears, Finn \& Steinmetz, 1994) y Alzheimer (Woodruff-Pak, Romano \& Papka, 1996).

Pese al entusiasmo que han despertado estos hallazgos, los estudios de condicionamiento con humanos distan mucho de la sofisticación que tiene el paradigma con conejos. Específicamente, si bien el paradigma de condicionamiento palpebral en humanos tiene una larga historia, la forma en que se utiliza en la actualidad adolece de muchas carencias metodológicas (Woodruff-Pak \& Steinmetz, 2002). En primer lugar, los humanos parpadean con mucha más frecuencia que los conejos y lo hacen por varias razones, no solo reflejas sino que también cognitivas de carácter voluntario (Spence \& Ross, 1959). Esto demanda que los protocolos de condicionamiento establezcan distinciones entre distintos tipos de respuesta, ya que no todas las respuestas de parpadeo que emiten las personas en presencia de un estímulo condicionado son, en estricto rigor, respuestas condicionadas. La omisión de estas consideraciones en varios estudios de condicionamiento en humanos puede explicar la inconsistencia de muchos resultados experimentales.

Por otra parte, la investigación con conejos ha puesto en evidencia que la generación de la respuesta condicionada no es la única consecuencia que resulta del establecimiento de una asociación entre el EC y el EI. Numerosos estudios han demostrado que el EC interfiere con la capacidad del EI para auto-generar su propio procesamiento. Una forma en la que esta interferencia puede ocurrir se relaciona con un fenómeno conocido como "Disminución Condicionada de la Respuesta Incondicionada" (DCRI, Kimble \& Ost, 1961), consistente en una reducción en la magnitud de la respuesta incondicionada cuando el EI es precedido por el EC.

El término DCRI fue originalmente adoptado por Kimble y Ost (1961), quienes evaluaron distintos intervalos entre estímulos y concluyeron que existía un intervalo óptimo con el cual era posible observar RC más robustas y RI disminuidas, siempre y cuando el EI fuese precedido por un EC. Hipotetizaron que esta disminución en la respuesta de parpadeo estaba controlada por el estímulo y sería una forma de inhibición condicionada. Desde entonces, el fenómeno ha sido observado en distintas especies y situaciones experimentales, tales como la respuesta galvánica de la piel en humanos (Baxter, 1966; Kimmel \& Pennypacker, 1962; Marcos \& Redondo, 2002; Redondo \& Mar- 
cos, 2000), la respuesta de parpadeo en conejos y humanos (Canli, Detmer \& Donegan, 1992; Canli \& Donegan, 1995; Hupka, Kwaterski \& Moore, 1970) y en la respuesta de aceleración cardíaca en humanos (Marcos \& Redondo, 2001; Redondo \& Marcos, 2003) y perros (Fitzgerald, 1966).

Wagner y sus colaboradores (Wagner, 1976, 1978, 1981; Wagner \& Vogel, 2010) sostienen que la Disminución Condicionada de la Respuesta Incondicionada (DCRI) es un fenómeno de priming, según el cual los estímulos predichos o anticipados tienden a ser procesados con menor efectividad que cuando son inesperados. En el condicionamiento clásico el EI pierde efectividad debido a que es anticipado o predicho por el EC. De acuerdo con este enfoque, el EC genera el pre-procesamiento del EI y contribuye a su pérdida de efectividad al momento de la estimulación.

Existen al menos cuatro estudios que demuestran diferentes vías por las cuales hay una disminución del procesamiento de un EI esperado. Terry y Wagner (1975) demostraron que un EI anticipado fue menos retenido en la memoria sobre un intervalo de trazo comparado con un EI no predicho por un estímulo discriminativo. Wagner, Rudy y Whitlow (1973) demostraron que un EI esperado fue menos efectivo como distractor que un EI inesperado. Un tercer hallazgo se deriva de las variaciones del efecto de bloqueo propuesto por Kamin (1969), quien demostró que un EI esperado pierde efectividad como reforzador de otro EC.

La cuarta demostración es un ejemplo típico de la DCRI y puede encontrarse en una serie de experimentos realizados por Donegan (1981) en el condicionamiento de parpadeo en conejos. Estos experimentos comienzan con una fase de entrenamiento discriminativo con un EC correlacionado con la presencia del EI (EC+) y otro EC correlacionado con su ausencia (EC-). Posteriormente, en una fase de prueba, se evalúa la amplitud de la RI en ensayos en los cuales el EI es precedido por un $\mathrm{EC}+\mathrm{o}$ por un EC-. Los resultados indicaron que la respuesta incondicionada fue significativamente menor en los ensayos precedidos por $\mathrm{EC}+$ que por EC-. Esto se debe a que la respuesta condicionada al estímulo EC+ fue un cierre del párpado anticipatorio, que imita a la RI y termina por sumarse con la respuesta generada por el EI. Adicionalmente, Donegan (1981) probó a sus animales con intensidades más bajas del EI que en el entrenamiento. Los resultados de la fase de prueba demostraron que a intensidades más bajas el EC+ facilita la respuesta de parpadeo al momento del EI, en cambio a una mayor intensidad el EC+ disminuye la respuesta de parpadeo.

A partir de sus resultados, Donegan (1981) concluyó que el EC tenía dos formas opuestas de influir en la magnitud de la RI. La primera tendencia de carácter aumentativo se presentó cuando los estímulos fueron probados con un EI de baja intensidad y la segunda de carácter diminutivo fue evidente con un EI de alta intensidad. Naturalmente ambos efectos actuarían simultáneamente, lo cual hace que las condiciones en que se observa priming de uno u otro requieran de diseños experimentales extremadamente sensibles.

En consecuencia, el presente estudio pretende demostrar estas influencias asociativas del EC sobre la RI, a partir de la estrategia experimental propuesta por Donegan (1981), pero utilizando el procedimiento de condicionamiento palpebral en humanos. Esta consiste en un entrenamiento de condicionamiento discriminativo en el cual se presenta un estímulo emparejado con el estímulo incondicionado (EC+) y uno no emparejado (EC-), que en una posterior fase de prueba, son ensayados en condiciones de alta y baja intensidad del estímulo incondicionado. De acuerdo con los hallazgos de Donegan (1981), se espera que la magnitud de la respuesta incondicionada a un EI de alta intensidad sea menor cuando el EI es precedido por el estímulo $\mathrm{EC}+$ que cuando es precedido por el estímulo EC-. Por otra parte, se espera que la magnitud de la respuesta incondicionada a un EI de baja intensidad sea mayor cuando el EI es precedido por el estímulo $\mathrm{EC}+$ que cuando es precedido por el estímulo EC-. 


\section{Método}

\section{Participantes}

Un total de 17 estudiantes de la Universidad de Talca participaron voluntariamente en el estudio, correspondientes a nueve hombres y ocho mujeres cuyas edades fluctuaron entre los 19 a 24 años. Estos firmaron un consentimiento informado aprobado por el Comité de Ética de la Universidad de Talca.

\section{Aparatos}

Las sesiones experimentales se realizaron en una habitación acústicamente aislada $(2.5 \times 2.8$ x 2.4 metros) y tenuemente iluminada mediante lámparas fluorescentes de 18 watts, ubicada en el laboratorio de Psicología de la Universidad de Talca. Los participantes ingresaron a la habitación y se sentaron en un sillón. El sistema Eyeblink Conditioning System (San Diego Instruments Inc., San Diego, CA) fue utilizado para configurar la sesión de entrenamiento, la presentación de estímulos y la medición de la amplitud de la respuesta palpebral.

Los participantes del estudio recibieron como estímulo incondicionado, uno táctil consistente en un soplo de aire en el contorno del ojo generado por un compresor de aire calibrado en intensidad mediante un medidor de presión y administrado por medio de un cintillo con un tubo de aire, cuya intensidad fue de 9 libras por pulgada cuadrada (psi) con una duración de 100 milisegundos. La intensidad del soplo de aire se describe en términos de presión, definido como la cantidad de fuerza por unidad de área de presión, la cual es reportada en psi. Como estímulos condicionados, un tono de 65 $\mathrm{dB}$ administrado por medio de audífonos MAICO y una vibración de $30 \mathrm{~Hz}$ con una duración de 500 milisegundos en cada caso. Respecto de la vibración, esta fue aplicada por medio de un masajeador instalado en el sillón en el cual se sentaban los participantes.
La respuesta palpebral fue medida por medio de un sensor infrarrojo colocado frente al ojo derecho de los participantes y sujetado por un cintillo que permitía mantener fija la posición del sensor independiente de los cambios posturales. La respuesta de parpadeo produce una interrupción en la emisión infrarroja, la que se traduce en cambios en el voltaje transmitido por el sensor. Se registró el valor de voltaje con una frecuencia de 1 muestra por cada 1 milisegundo. Con el fin de tener una medida de una respuesta de parpadeo típica, se realizó un ensayo de estandarización con un voluntario no entrenado. El valor de voltaje máximo obtenido con este participante fue considerado como una respuesta de amplitud $100 \%$. Las respuestas máximas de cada participante durante el ensayo fueron expresadas como un porcentaje de este valor.

Durante los 200 milisegundos que anteceden a la presentación del EC se registraron los voltajes en ausencia de estimulación (línea base). El periodo correspondiente a las respuestas alfa $(\alpha)$ abarcó desde el momento de la aparición del EC hasta los 300 milisegundos, por su parte, la respuesta condicionada (RC) fue registrada desde los 300 milisegundos desde la aparición del EC hasta la presentación del EI (600 milisegundos) y la respuesta incondicionada (RI) fue medida desde la aparición del EI hasta los 800 milisegundos del ensayo. En los ensayos, en los cuales se presentó el EC emparejado con el EI (EC+) se consideró un intervalo entre estímulos de 400 milisegundos.

Se consideró como un ensayo válido, aquella respuesta palpebral con una amplitud inferior al $20 \%$ obtenido, en ausencia de estimulación (línea base) y una amplitud inferior al $5 \%$ durante el periodo de la respuesta alfa. Por su parte, en los ensayos válidos, se consideró como respuesta condicionada la que superó una amplitud del 5\% en el periodo correspondiente. De igual modo, se consideró como respuesta incondicionada toda respuesta que superó una amplitud del $5 \%$ en el periodo correspondiente. 


\section{Procedimiento}

La sesión experimental consideró tres etapas: preparación, adaptación y entrenamiento-prueba. La tarea en conjunto tuvo una duración de 90 minutos aproximadamente. En la etapa de preparación, los participantes leyeron y firmaron un consentimiento informado y tuvieron la posibilidad de consultar sobre cualquier aspecto comprometido en el experimento. Posteriormente, el experimentador colocó los audífonos y el cintillo con el sensor infrarrojo y tubo de aire en la cabeza de los participantes. En ese momento, se les solicitó a los participantes que parpadearan repetidamente con el fin de obtener una respuesta de parpadeo detectable. Además, se les dio la instrucción de prestar atención a la película Gold Rush, de 90 minutos de duración aproximadamente, enfatizando que, luego de finalizada la sesión experimental, se les preguntaría sobre esta. Con esto se pretendió controlar la incidencia de respuestas voluntarias que pudiesen contaminar la medición fidedigna de la respuesta condicionada (Clark \& Squire, 1999). En la etapa de adaptación, el experimentador cerró la cámara experimental y los participantes fueron sometidos a un periodo de 5 minutos sin estimulación, con el fin de que pudiesen adaptarse al contexto, al dar inicio a la presentación de la película. Luego, se ejecutó la siguiente fase desde el computador.

En la etapa de entrenamiento-prueba los participantes recibieron primero un total de 132 ensayos, de los cuales 66 ensayos correspondían al $\mathrm{EC}+$ que co-termina con el EI, entremezclados con 66 del estímulo EC- por sí solo. El intervalo entre ensayos varió aleatoriamente entre 15, 20 y 25 segundos. Al finalizar este entrenamiento, los participantes recibieron una serie de ensayos de prueba, en los que se examinó la RI a estímulos incondicionados de alta (9 psi) y baja (3 psi) intensidad cuando eran precedidos por el $\mathrm{EC}+$ o EC-. Cada tipo de ensayo de prueba se repitió 8 veces, en total fueron 32 ensayos de prueba. De esta manera, la fase de entrenamiento-prueba contó con un total de 132 ensayos de entrenamiento (66 ensayos de EC+ y 66 ensayos de EC-) y 32 ensayos de prueba (8 de cada tipo). Tanto los ensayos de entrenamiento como los de prueba fueron presentados en orden pseudo-aleatorio, el cual varió entre los participantes. Para 9 de los participantes el estímulo EC+ fue el tono y el estímulo EC- la vibración, mientras que para los 8 restantes, la naturaleza de los estímulos se invirtió (es decir EC+ = Vibración y EC- = Tono). Una vez finalizada la fase de entrenamiento-prueba se formularon las preguntas sobre la película.

\section{Análisis estadísticos}

Con el fin de examinar si hubo condicionamiento producto de la asociación de los estímulos y no a la mera exposición al estímulo incondicionado, los 66 ensayos de condicionamiento con cada estímulo se agruparon en 6 bloques de 11 ensayos en los cuales se calculó la amplitud de la respuesta condicionada. Para evaluar la significación estadística de los efectos del experimento se realizó un ANOVA de medidas repetidas de 2 (Estímulos: EC+y EC-) X 6 (Bloque de entrenamiento: bloque 1, bloque 2, ..., bloque 6) utilizando la amplitud de la respuesta condicionada de parpadeo como variable dependiente. Si la interacción estímulo x bloque resulta significativa, se examinan los efectos simples de la variable estímulo en cada bloque con la prueba post-hoc Least Significant Difference (LSD).

Por otro lado, con el fin de examinar los efectos del condicionamiento sobre la respuesta incondicionada, se calculó la amplitud promedio de la respuesta incondicionada en los ensayos de prueba. La significación estadística de los efectos de interés se examinó por medio de un ANOVA de medidas repetidas de 2 (Estímulo: EC+ y EC-) X 2 (Intensidad del EI: baja y alta) utilizando la amplitud de la respuesta incondicionada de parpadeo como variable dependiente. Si la interacción estímulo $\mathrm{x}$ intensidad resulta significativa, se examinan los efectos simples de la variable estímulo en cada intensidad con la prueba post-hoc LSD. 


\section{Resultados}

La figura 1 presenta la amplitud media de la respuesta condicionada de parpadeo según los estímulos (EC+ y EC-) en el curso del entrenamiento. Se observa que el estímulo emparejado con el estímulo incondicionado (EC+) muestra cambios progresivos de la amplitud de la respuesta condicionada, que aumenta hasta alcanzar asíntota. Por el contrario, se observa que el estímulo que se presentó por sí solo (EC-) inicialmente aumenta en amplitud de la respuesta condicionada, probablemente debido a la generalización de EC+ a EC-, pero posteriormente disminuye progresivamente lográndose la discriminación.

El ANOVA de medidas repetidas arroja un efecto principal de bloque de entrenamiento, $F(5,80)$ $=3.357 ; p=.008 ; \eta^{2}$ parcial $=.173$, y estímulos, $F(1,16)=6.131 ; p=.025 ; \eta^{2}$ parcial $\left.=.277\right), \mathrm{y}$ efecto de interacción entre bloque y estímulos, $F(5$, $80)=3.314 ; p=.009 ; \eta^{2}$ parcial $\left.=.172\right)$. El efecto bloque revela que existen cambios progresivos de la amplitud de la respuesta condicionada en el curso del entrenamiento y el efecto estímulos, que la amplitud de la respuesta condicionada es mayor al estímulo EC+ que a EC-. El efecto de interacción estímulos $\mathrm{x}$ bloques revela que en determinados bloques del entrenamiento existen diferencias en la amplitud de la respuesta condicionada entre los estímulos EC+ y EC-.

Dado que la interacción estímulos x bloques fue significativa, se examinaron los efectos simples, los cuales revelaron que en el primer bloque ( $p=$ .692) no existen diferencias en la amplitud de la respuesta condicionada al estímulo EC+ en comparación con el EC-. En el segundo bloque existen diferencias marginalmente significativas $(p=.079)$ y en los restantes bloques existen diferencias estadísticamente significativas que evidencian una mayor respuesta condicionada al estímulo $\mathrm{EC}+$ que a EC- $(p s<.028)$.

Debido a que hubo condicionamiento discriminativo de la $\mathrm{RC}$, se concluye que se produjo una asociación entre el EC y el EI que no se debe a la

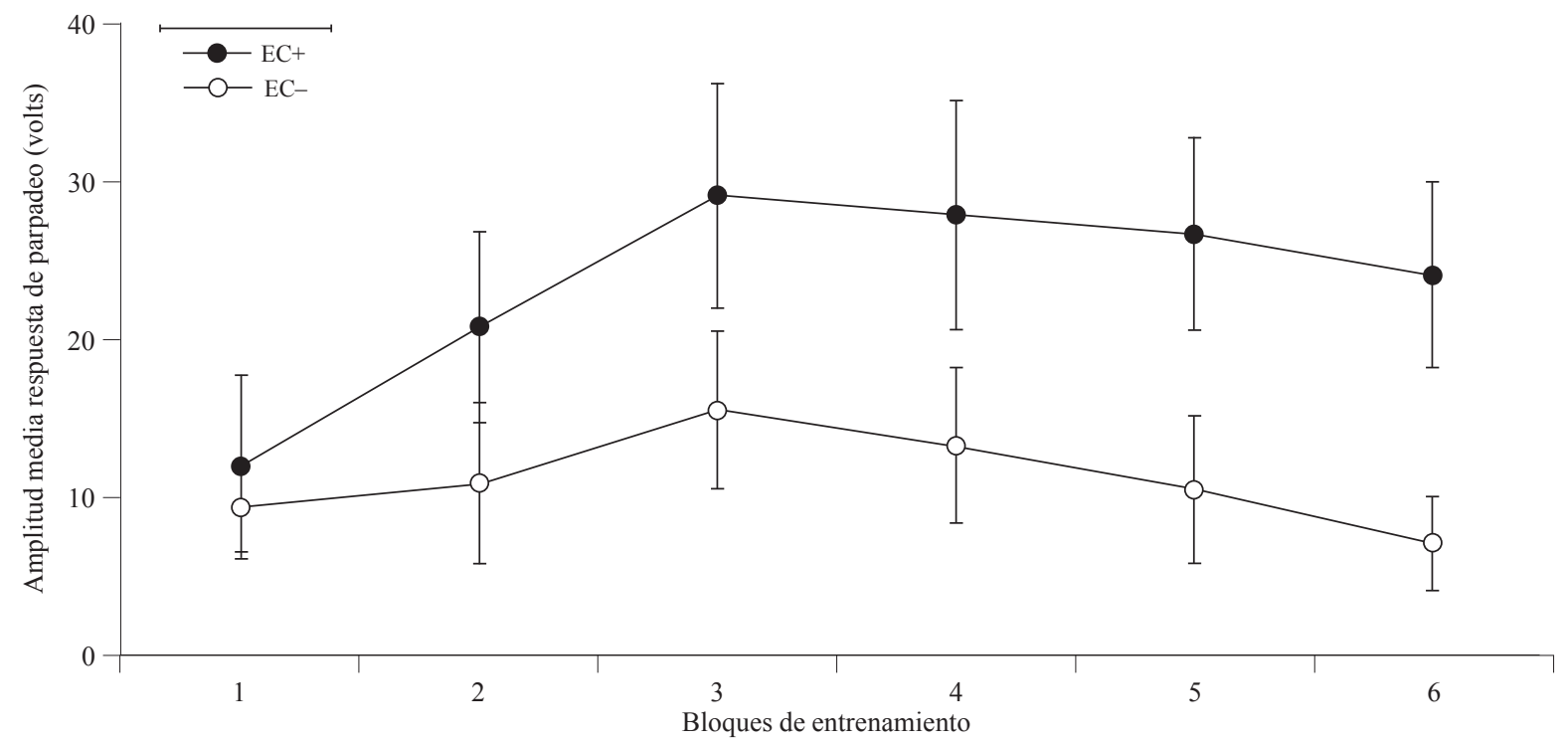

Figura 1. Amplitud media de la respuesta condicionada de parpadeo según el estímulo entrenado (EC+ y EC-) en el curso del entrenamiento

Nota: Las barras de error representan el error estándar de la media. 
mera exposición de los estímulos condicionados e incondicionados o pseudo-condicionamiento (Rescorla, 1967).

Los datos de mayor interés se presentan en la figura 2, la cual muestra la amplitud de la RI en los distintos tipos de ensayos de prueba para cada estímulo entrenado (EC+ y EC-). Se observa una menor amplitud de la respuesta incondicionada al estímulo EC+ en comparación con el EC- cuando son probados con un EI de alta intensidad (9 psi), mientras que cuando son probados con un EI de baja intensidad (3 psi), se observa una mayor amplitud de la respuesta incondicionada al estímulo EC+ en comparación con el EC-.

El ANOVA de medidas repetidas de 2 (Estímulo: EC+ y EC-) X 2 (Intensidad del EI: baja y alta) arrojó efecto principal de intensidad, $F(1,16)=$ $17.569 ; p=.001 ; \eta^{2}$ parcial $=.523$, pero no hubo efecto principal de estímulos $(p=.405)$. Además, reveló el efecto de interacción entre estímulos e intensidad, $F(1,16)=11.630 ; p=.004 ; \eta^{2}$ parcial $=.421$. El efecto intensidad revela que hubo una mayor amplitud de la respuesta incondicionada a un EI de alta intensidad (9 psi) en comparación con uno de baja intensidad (3 psi), por su parte, la ausencia del efecto estímulos, indica que no existen diferencias en la amplitud de la respuesta incondicionada al estímulo EC+ en comparación con EC-. El efecto de interacción entre estímulos $\mathrm{x}$ intensidad revela que existen diferencias en la amplitud de la respuesta incondicionada que varían según el estímulo y la intensidad del EI con el cual fueron probados los participantes del estudio.

Dado que la interacción fue significativa se examinaron los efectos simples, los cuales confirman las observaciones ya que revelaron que hubo una mayor amplitud de la respuesta incondicionada al estímulo EC+ en comparación a EC- cuando fueron probados con un EI de baja intensidad $(p=.012)$, mientras que cuando fueron probados con un EI de alta intensidad, contrario a lo esperado, no hubo diferencias estadísticamente significativas entre los estímulos ( $p=.167)$.

En conclusión, en el presente estudio se demostró que el EC adquiere la propiedad para producir una RC, sin embargo, no existen diferencias esta-

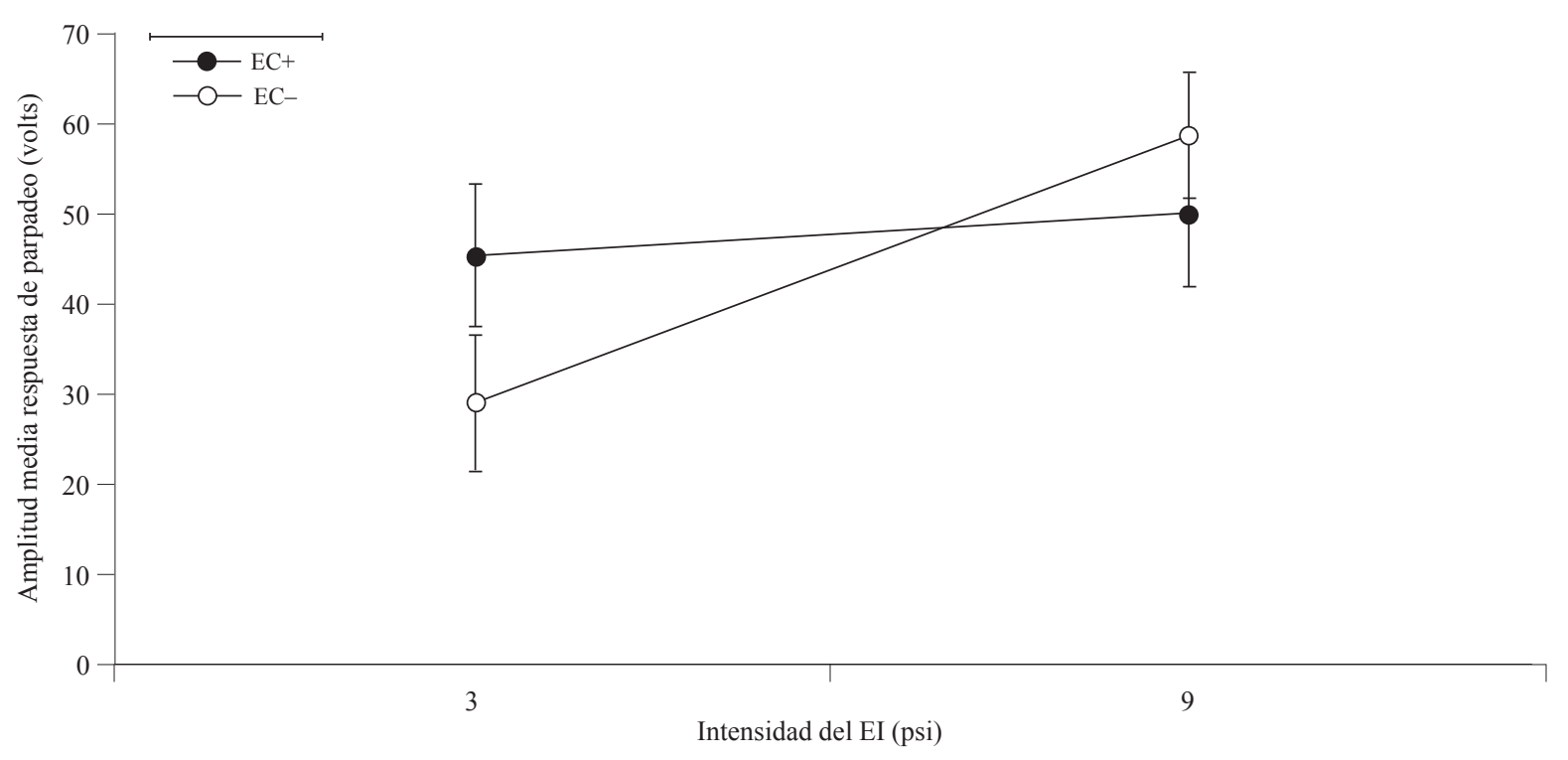

Figura 2. Amplitud media de la respuesta incondicionada de parpadeo según los estímulos (EC+y EC-) probados con un estímulo incondicionado de baja y alta intensidad (3 y 9 psi respectivamente)

Nota: Las barras de error representan el error estándar de la media. 
dísticamente significativas en la magnitud de la RI cuando el EC es presentado en conjunto con un EI de alta intensidad.

\section{Discusión}

Los resultados de la presente investigación no son concluyentes para determinar que un estímulo capaz de desarrollar una respuesta condicionada interfiere en la generación de la respuesta incondicionada del estímulo incondicionado. En primer lugar, el resultado más evidente del proceso de condicionamiento palpebral es la generación por parte del EC de una respuesta condicionada de parpadeo anticipatoria, que termina por sumarse con la respuesta generada por el EI cuando este ocurre. Esto se observó en la fase de prueba, donde la amplitud de la RI fue mayor al estímulo EC+ que a EC- cuando ambos fueron emparejados con un EI de baja intensidad. En segundo lugar, a diferencia del estudio de Donegan (1981) no existe evidencia concluyente para determinar que el EC adquiere la propiedad de generar una disminución en el procesamiento del EI a medida que progresa el aprendizaje, puesto que en la fase de prueba, contrario a lo esperado, no hubo diferencias en la amplitud de la RI entre los estímulos condicionados presentados en conjunto con un EI de alta intensidad.

El procedimiento seguido por Donegan (1981) y por la presente investigación permite descartar explicaciones alternativas con las cuales se podría cuestionar si el efecto de facilitación observado es producto del condicionamiento. Por ejemplo, se ha demostrado que la mera repetición de un estímulo biológicamente significativo y con valencia emocional, como lo es el soplo de aire en el ojo, puede producir un estado de sensibilización que conduce a un aumento generalizado en los niveles de respuesta a cualquier estímulo. Si este fuera el caso, el aumento en las respuestas de parpadeo a un estímulo condicionado podría ser el resultado de lo que se ha denominado pseudo-condicionamiento (Rescorla, 1967). Sin embargo, esta posibilidad fue controlada a partir de un entrenamiento discriminativo en el cual un estímulo condicionado se presenta en conjunto con el estímulo incondicionado $(\mathrm{EC}+)$ mientras que otro estímulo se presenta por sí solo (EC-). En este procedimiento, el estímulo EC- recibe exactamente el mismo tratamiento que $\mathrm{EC}+$, excepto que se presente explícitamente no pareado con el EI. De este modo, en caso de existir condicionamiento, esto debería reflejarse en una mayor frecuencia y amplitud de las respuestas de parpadeo provocadas por el estímulo $\mathrm{EC}+$ que las provocadas por el estímulo EC-, tal como se demostró en el estudio de Donegan (1981) y en la presente investigación.

Por otra parte, es necesario reconocer que una de las complicaciones que surgen al analizar cualquier tendencia a la disminución de la respuesta incondicionada de un estímulo aversivo, como lo son generalmente los estímulos incondicionados, es que el EC que se asocia con este puede producir una respuesta emocional que potencia una amplia gama de conductas defensivas (Bombace, Brandon \& Wagner, 1991; Brandon, Bombace, Falls \& Wagner, 1991; Brandon \& Wagner, 1991). Por ejemplo, Brandon, Betts y Wagner (1994) demostraron que los estímulos condicionados, tanto explícitos como contextuales, que se asocian con estímulos incondicionados aversivos, pueden actuar como potenciadores de otras respuestas reflejas que no se habían considerado en los episodios de condicionamiento. Sin embargo, esto no se puede observar debido a que ambas tendencias se confunden.

De acuerdo con esto, la presencia de estas respuestas emocionales generalizadas pudo complicar la observación del fenómeno de la DCRI en el presente estudio, ya que una aparente ausencia de disminución puede ser el resultado de una combinación entre potenciación y disminución. Por ejemplo, Brandon, Bell y Wagner (Wagner \& Vogel, 2010), sirviéndose del hecho de que la respuesta condicionada de parpadeo en conejos es altamente lateralizada, idearon una estrategia experimental para separar estos efectos. Con esta 
ventaja, es posible evaluar la DCRI, no solo comparando la respuesta al EI cuando es precedido por el EC cuando no es precedido por el EC, sino que también cuando el EI es precedido por un EC que fue asociado con un EI sensorialmente diferente (por ejemplo el otro ojo) pero que genera las mismas respuestas emocionales generalizadas que el otro EC. El experimento constó de una fase inicial, en la cual un EC D fue emparejado con un EI táctil aplicado en el ojo derecho (D+), otro EC I fue emparejado con el mismo EI pero aplicado en el ojo izquierdo ( $\mathrm{I}+$ ) y un tercer estímulo $X$ fue presentado por sí solo. Posteriormente, se realizó una serie de pruebas destinadas a comparar la magnitud de la respuesta incondicionada cuando el EI era precedido por el mismo EC usado en el entrenamiento, el EC alternativo y el EC neutral. El primer hallazgo replica los resultados típicos de DCRI, ya que las respuestas al EI fueron menores cuando este era precedido por el mismo estímulo con el cual se hizo el emparejamiento $(D$ o $I$ dependiendo del ojo de aplicación del EI en la prueba) que cuando era precedido por el estímulo neutral $X$. Otro hallazgo de este estudio fue que esta diferencia contiene una subestimación de la DCRI. Esto último, porque la amplitud de la RI cuando el EI es precedido por el estímulo que fue emparejado con el ojo alternativo fue mayor que cuando era precedido por el estímulo neutral. Con este último resultado, uno podría suponer que existe una respuesta emocional condicionada que potencia la RI sensorial. Para que esta potenciación de la RI ocurra, basta con la presentación de cualquier EC, independientemente de la concordancia en la localización del EI entre el entrenamiento y la prueba. Naturalmente, cuando se prueba el mismo EI utilizado en el entrenamiento, los efectos emocionales potenciadores se oponen a los efectos decrementales sensoriales producidos por el EC.

Brandon et al. (Wagner \& Vogel, 2010) realizaron otros experimentos similares pero utilizando ECs progresivamente más largos con el fin de evaluar si estos efectos se repiten con estímulos del tipo contextual. Nuevamente se obtuvieron tendencias potenciadoras generalizadas causadas por los estímulos del tipo contextual pero no siempre hubo evidencia de DCRI al comparar las respuestas al EI precedido por el EC aplicado al mismo ojo con las respuestas al EI precedido por el EC neutral. Sin embargo, la potenciación fue consistentemente menor cuando el EI era precedido por el EC aplicado al mismo ojo que al ojo alterno, lo cual estaría proporcionando evidencia indirecta de disminución.

Wagner y Vogel (2010) esbozaron una explicación cuantitativa de este dilema basada en los modelos SOP (Wagner, 1981) y su extensión AESOP (Wagner \& Brandon, 1989), según la cual las respuestas condicionadas e incondicionadas sensoriales pueden ser influenciadas por asociaciones emotivas. Los supuestos son los siguientes:

1. Cada vez que se presenta conjuntamente un estímulo conductualmente neutral, por ejemplo, un tono de baja intensidad y un estímulo biológicamente significativo, como un fuerte soplo de aire en el contorno del ojo, se establece una asociación entre el tono, que actuaría como estímulo condicionado (EC) y el soplo de aire que actuaría como estímulo incondicionado (EI).

2. Según el modelo SOP, tanto el EC como el EI activan un par de unidades de procesamiento: una unidad primaria seguida por una unidad secundaria que recurrentemente inhibe a la primaria. Una vez que se presenta el estímulo correspondiente (tono o soplo de aire) y durante cada momento de su duración, la unidad primaria respectiva es activada, lo cual provoca la respuesta y la activación de la unidad secundaria respectiva. Una vez que es activada, la unidad secundaria recurrentemente inhibe a la primaria, dejándola temporalmente menos susceptible de ser activada por el estímulo. Con el paso del tiempo, la actividad secundaria decae, liberándose a la unidad primaria de la inhibición. 
3. El modelo asume que el EC y el EI desarrollan una asociación excitatoria en la medida que el grado de activación simultánea de sus respectivas unidades primarias es sustancialmente más grande que el grado de activación primaria del EC coincidente con la activación secundaria del EI.

4. Si la asociación que se forma entre el EC (tono) y el EI (soplo de aire) es excitatoria, entonces el tono adquiere la capacidad, por medio de su actividad primaria, para generar asociativamente actividad secundaria en el soplo de aire, aún cuando este último no se encuentre presente. Según esto, la respuesta condicionada (RC) es una función del grado de actividad primaria del tono, y de la fuerza de la conexión entre esta unidad y la actividad secundaria del soplo de aire.

De acuerdo con este mecanismo, el modelo SOP explicaría la facilitación condicionada como una respuesta condicionada anticipatoria que termina por sumarse a la respuesta incondicionada. Esta tendencia es más notoria cuando en la fase de prueba se presenta un EI de intensidad menor (3 psi) que aquella que se utilizó en el entrenamiento, ya que la respuesta condicionada sería mayor que la incondicionada producida por el EI débil. Dado que la actividad secundaria del soplo de aire inhibe recurrentemente su propia actividad primaria, la presencia del tono produce una disminución en la respuesta incondicionada (RI) del soplo de aire (Donegan \& Wagner, 1987). Esta tendencia es más notoria cuando se utiliza un EI de alta intensidad (9 psi), puesto que es más fácil observar decrementos cuando la RI se encuentra a niveles elevados. Hasta este punto, la predicción del modelo es que la DCRI es una consecuencia del condicionamiento. Con el fin de explicar el hecho de que existan respuestas emocionales que pudieron oscurecer la observación de la DCRI en el presente estudio Wagner y Vogel (2010) apelan a los siguientes supuestos adicionales:
1. Ya que el mismo estímulo incondicionado, por ejemplo un fuerte soplo de aire en el contorno del ojo es capaz de provocar una respuesta de parpadeo y, al mismo tiempo, una respuesta emocional como el miedo, el modelo debe asumir que la presentación del estímulo provoca la activación de dos pares de unidades de procesamiento, una para cada respuesta. Esto es consistente con lo planteado por Wagner y Brandon (1989), quienes señalan que los estímulos incondicionados deberían representarse por medio de unidades sensoriales y emotivas, donde cada una establecería asociaciones independientes con el estímulo condicionado (tono).

2. Se asume que aunque la asociación del tono con las respuestas sensorial y emotiva sigue las mismas reglas de SOP, hay importantes diferencias paramétricas en el curso temporal de ambas respuestas. Wagner y Vogel (2010) enfatizaron que la diferencia más notable es que el procesamiento emotivo es mucho más lento que el procesamiento sensorial, lo cual le otorga una mayor duración a la actividad primaria emotiva y, por consiguiente, una mayor probabilidad de asociación con estímulos condicionados de larga duración (o estímulos designados como contextuales).

De acuerdo con este análisis, es factible esperar que la DCRI pudiera ser oscurecida por los efectos emocionales que potencian las respuestas sensoriales controladas por un mismo estímulo. A partir de los datos de Brandon et al. (Wagner \& Vogel, 2010), resulta natural especular que aquellos estudios que no han observado disminución de la respuesta incondicionada no hayan sido lo suficientemente sensitivos para separar estos efectos decrementales y potenciadores, y además no hayan considerado la posibilidad de que esos efectos pueden existir aun cuando el resultado conductual final sea una repuesta potenciada. Esto invita a evaluar más sistemáticamente los fenómenos y diseños de los 
experimentos con resultados negativos como en el presente estudio.

\section{Referencias}

Baxter, R. (1966). Diminution and recovery of the UCR in delayed and trace classical GSR conditioning. Journal of Experimental Psychology, 71(3), 447-451. doi: 10.1037/h0022977

Bombace, J., Brandon, S., \& Wagner, A. (1991). Modulation of a conditioned eyeblink response by a putative emotive stimulus conditioned with hindleg shock. Journal of Experimental Psychology: Animal Behavior Processes, 17(3), 323-333. doi: 10.1037/0097-7403.17.3.323

Bracha, V., Zhao, L., Wunderlich, D., Morrissy, S., \& Bloedel, J. (1997). Patients with cerebellar lesions cannot acquire but are able to retain conditioned eyeblink reflexes. Brain, 120, 14011413. doi: 10.1093/brain/120.8.1401

Brandon, S., Betts, S., \& Wagner, A. (1994). Discriminated lateralized eyeblink conditioning in the rabbit: An experimental context for separating specific and general associative influences. Journal of Experimental Psychology: Animal Behavior Processes, 20, 292-307. doi: 10.1037/0097-7403.20.3.292

Brandon, S., Bombace, J., Falls, W., \& Wagner, A. (1991). Modulation of unconditioned defensive reflexes via an emotive Pavlovian conditioned stimulus. Journal of Experimental Psychology: Animal Behavior Processes, 17, 312-322. doi: 10.1037/0097-7403.17.3.312

Brandon, S., \& Wagner, A. (1991). Modulation of a discrete Pavlovian conditioning reflex by a putative emotive Pavlovian conditioned stimulus. Journal of Experimental Psychology: Animal Behavior Processes, 17(3), 299-311. doi: 10.1037/0097-7403.17.3.299

Canli, T., Detmer, W., \& Donegan, N. (1992). Potentiation or diminution of discrete motor unconditioned responses (rabbit eyeblink) to an aversive pavlovian unconditioned stimulus by two asso- ciative processes: Conditioned fear and conditioned diminution of unconditioned stimulus processing. Behavioral Neuroscience, 106(3), 498-508. doi: 10.1037/0735-7044.106.3.498

Canli, T., \& Donegan, N. (1995). Conditioned diminution of the unconditioned response in rabbit eyeblink conditioning: Identifying neural substrates in the cerebellum and brainstem. Behavioral Neuroscience, 109(5), 874-892. doi: 10.1037/0735-7044.109.5.874

Clark, R., \& Squire, L. (1999). Human eyeblink classical conditioning: Effects of manipulating awareness of the stimulus contingencies. Psychological science, 10(1), 14-18. doi: 10.1111/1467-9280.00099

Christian, K., \& Thompson, R. (2003). Neural substrates of eyeblink conditioning: Acquisition and retention. Learning \& Memory (Cold Spring Harbor, N.Y.), 10, 427-455. doi: 10.1101/ $\operatorname{lm} .59603$

Daum, I., Schugens, M., Ackermann, H., Lutzenberger, W., Dichgans, J., \& Birbaunmer (1993). Classical conditioning after cerebellar lesions in humans. Behavioral Neuroscience, 5, 748-756. doi: 10.1037/0735-7044.107.5.748

Donegan, N. (1981). Priming-produced facilitation or diminution of responding to a Pavlovian unconditioned stimulus. Journal of Experimental Psychology: Animal Behavior Processes, 7, 295-312. doi: 10.1037/0097-7403.7.4.295

Donegan, N., \& Wagner, A. (1987). Conditioned diminution and facilitation of the UR: A sometimes opponent-process interpretation. En I. Gormezano, W. Prokasy, \& R. Thompson (Eds.), Classical Conditioning (pp. 339-369). Hillsdale, NJ: Erlbaum.

Fanselow, M., \& LeDoux, J. (1999). Why we think plasticity underlying Pavlovian fear conditioning occurs in the basolateral amygdala. Neuron, 23, 229-232. doi: 10.1016/S08966273(00)80775-8

Fitzgerald, R. D. (1966). Some effects of partial reinforcement with shock on classically condi- 
tioned heart rate in dogs. American Journal of Psychology, 79, 242-249. doi: 10.2307/1421130 Gormezano, I., Schneiderman, N., Deux, E., \& Fuentes, I. (1962). Nictitating membrane: Classical conditioning and extinction in the albino rabbit. Science, 138(3536), 33-34. doi: 10.1126/science. 138.3536 .33

Hupka, R., Kwaterski, S., \& Moore, J. (1970). Conditioned diminution of the UCR: differences between the human eyeblink and the rabbit nictitating membrane response. Journal of Experimental Psychology, 85(1), 45-51. doi: 10.1037/ h0028584

Kamin, L. J. (1969). Predictability, surprise, attention and conditioning. En B. A. Campbell \& R.M. Church (Eds.), Punishment and aversive behavior (pp. 279-296). New York: Appleton Century Crofts.

Kimble, G., \& Ost, J. (1961). A conditioned inhibitory process in eyelid conditioning. Journal of Experimental Psychology, 83, 45-51. doi: 10.1037/h0044932

Kimmel, H., \& Pennypacker, H. (1962). Conditioned diminution of the unconditioned response as a function of the number of reinforcements. Journal of Experimental Psychology, 64(1), 20-23. doi: 10.1037/h0045327

Lanuza, E., Moncho-Bogani, J., \& LeDoux, J. (2008). Unconditioned stimulus pathways to the amygdala: Effects of lesions of the posterior intralaminar thalamus on foot-shock-induced c-Fos expression in the subdivisions of the later amygdala. Neuroscience, 155, 959-968. doi: 10.1016/j.neuroscience.2008.06.028

Marcos, J., \& Redondo, J. (2001). Relation between conditioned stimulus-elicit responses and unconditioned response diminution in long-interval human heart-rate classical conditioning. The Spanish Journal of Psychology, 4(1), 11-18. doi: 10.1017/S1138741600005606.

Marcos, J., \& Redondo, J. (2002). Differential effects of expectancy and associative mechanisms on diminution of unconditioned response in elec- trodermal classical conditioning. Psicotehma, 14(2), 375-381. Recuperado de http://www. psicothema.com/pdf/735.pdf

Mauk, M., Medina, J., Nores, W., \& Ohyama, T. (2000). Cerebellar function: coordination, learning or timing? Current Biology, 10, 522-525. doi: 10.1016/S0960-9822(00)00584-4

Pavlov, I. (1927). Conditioned reflexes, (G.V. Anrep, Trans.). London: Oxford University Press.

Redondo, J., \& Marcos, J. (2000). Efecto de la interferencia de respuesta sobre la disminución de la respuesta electrodérmica incondicionada. Psicothema, 12(1), 125-129. Recuperado de http://www.psicothema.com/pdf/263.pdf

Redondo, J., \& Marcos, J. (2003). Effects of CSUS interval on unconditioned response diminution in human heart rate classical conditioning. Journal of Psychophysiology, 17, 30-38. doi: 10.1027//0269-8803.17.1.30

Rescorla, R. (1967). Pavlovian conditioning and its proper control procedures. Psychological Review, 74(1), 71-80. doi:10.1037/h0024109

Sanderson, D., Pearce, J., Kyd, R., \& Aggleton, J. (2006). The importance of the rat hippocampus for learning the structure of visual arrays. The European Journal of Neuroscience, 24, 17811788. doi: 10.1111/j.1460-9568.2006.05035.x

Schugens, M., \& Daum, I. (1999). Long-term retention of classical eyeblink conditioning in amnesia. NeuroReport, 10, 149-152. doi: 0.1097/00001756-199901180-00028

Sears, L., Finn, P., \& Steinmetz, J. (1994). Abnormal classical eye-blink conditioning in autism. Journal of Autism and Developmental Disorders, 24(6), 737-751. doi: 10.1007/BF02172283

Siegel, S. (1975). Evidence from rats that morphine tolerance is a learned response. Journal of Comparative and Physiological Psychology, 89(5), 468-506. doi: 10.1037/h0077058

Spence, K., \& Ross, L. (1959). A methodological study of the form and latency of eyelid responses in conditioning. Journal of Experimental Psychology, 58(5), 376-381. doi: 10.1037/h0045837 
Steinmetz, J., Tracy, J., \& Green, J. (2001). Classical eyeblink conditioning: Clinical models and applications. Integrative Physiological and Behavioral Science, 36(3), 220-238. doi: 10.1007/ BF02734095

Terry, W. S., \& Wagner, A. R. (1975). Short-term memory for "surprising" vs. "expected" unconditioned stimuli in Pavlovian conditioning. Journal of Experimental Psychology: Animal Behavior Processes, 1, 122-133. doi: 10.1037/0097-7403.1.2.122

Thompson, R., Thompson, J., Kim, J., Krupa, D., \& Shinkman, P. (1998). The nature of reinforcement in cerebellar learning. Neurobiology of Learning and Memory, 70, 150-176. doi: 10.1006/nlme.1998.3845

Wagner, A. R. (1976). Priming in STM: An information-processing mechanism for self-generated or retrieval-generated depression in performance. En T. J. Tighe, \& R. N. Leaton (Eds.), Habituation: Perspectives from child development, animal behavior, and neurophysiology (pp. 95128). Erlbaum: Nueva York.

Wagner, A. R. (1978). Expectancies and the priming of STM. En S. H. Hulse, H. Fowler, \& W. K. Honig (Eds.), Cognitive processes in animal behavior (pp. 177-209). Hillsdale, NJ: Erlbaum.

Wagner, A. R. (1981). SOP: A model of automatic memory processing in animal behavior. En N. E. Spear \& R. R. Miller (Eds.), Information processing in animals: Memory mechanisms (pp. 5-47). Hillsdale, NJ: Erlbaum.
Wagner, A., \& Brandon, S. (1989). Evolution of a structured connectionist model of Pavlovian conditioning (AESOP). En S., Klein, \& R., Mowrer (Eds), Contemporary Learning Theories: Pavlovian Conditioning and the Status of Traditional Learning Theory. Hillsdale, NJ: Erlbaum.

Wagner, A., Rudy, J., \& Whitlow, J. (1973). Rehearsal in animal conditioning. Journal of Experimental Psychology, 97, 407-426. doi: 10.1037/ h0034136

Wagner, A., \& Vogel, E. (2010). Associative modulation of US processing: implications for understanding of habituation. En Schmajuk, N. (Ed.). Computational Models of Conditioning (pp. 150-185). Durham: Duke University Medical Center.

Woodruff-Pak, D., Romano, S., \& Papka, M. (1996). Training to criterion in eyeblink classical conditioning in alzheimer's disease, down's syndrome with Alzheimer's disease, and Healthy Elderly. Behavioral Neuroscience, 110(1), 2229. doi: 10.1037/0735-7044.110.1.22

Woodruff-Pak, D., \& Steinmetz, J. (2002). Past, present, and future of human eyeblink classical conditioning. En D. Woodruff-Pak \& J. Steinmetz (Eds.), Eyeblink classical conditioning: Volume I, applications in humans (pp. 5-7). New York: Kluwer academic publishers.

\section{Fecha de recepción: 13 de marzo de 2014 fecha de aceptación: 13 de agosto de 2014}

